\title{
DIE LÖSUNG EINES PROBLEMS BEZÜGLICH DES DURCHSCHNITTES ZWEIER MODULARER RECHTSIDEALE IN EINEM RING
}

\author{
Von \\ F. SZÁSZ (Budapest)
}

In der Theorie des Jacobsonschen Radikales eines (assoziativen) Ringes spielen die mccularen maximalen Rechtsideale und die quasimodularen maximalen Rechtsid€ale eine wichtige Rolle. Das Jacobsonsche Radikal eines Ringes stimmt nämlich scwohl mit dem Durchschnitt aller modularen maximalen Rechtsideale als auch mit $c^{\prime} \in \mathrm{m}$ Durchechnitt aller quasimodularen maximalen Rechtsideale des Ringes üterein (vgl. Jaconson [1, Theorem 1.6.1 (1)], Kertész [2, Satz 5.24 (g)]).

Eekarntlich wird ein Rechtsideal $R$ eines Ringes $A$ modular (bzw. quasimodular) in $A$ gerannt, wenn es ein Element $a \in A$ mit $x-a x \in R$ für jedes $x \in A$ gibt (bzw. $R: A \subseteq R$ gilt, wotei $R: A=[y ; y \in A ; A y \subseteq R]$ ist). Offenbar ist jedes modulare Rechtsiceal auch quasimodular im Ring. Das Problem 3 des Buches [2] von Kertész löserd hat Verfasser [6] die Existenz eines Ringes mit einem quasimodularen maximalen, aker nicht modularen Rechtsideal gezeigt. ${ }^{1}$ Es soll bemerkt werden, daß nach JACOESON [1, Proposition 3. 6. 1 (2)] der Durchschnitt von endlich vielen modularen maximalen Rechtsidealen in einem Ring stets modular ist. Mit ähnlichen Methoden, wie JaCOBSON [1] gezeigt hat, hat Kertész [2, Satz 5. 2] bewiesen, daß der Durchschnitt $R_{1} \cap R_{2}$ zweier modularer Rechtsideale $R_{1}$ und $R_{2}$ des Ringes $A$ ebenfalls modular ist, wenn die Bedingung $R_{1}+R_{2}=A$ gilt. Diese Tatsachen sind bekanntlich für einen eleganten Beweis des Wedderburn-Artinschen Satzes über die Struktur der Ringe ohne von Null verschiedenes (Jacobsonsches) Radikal und mit Minimalbedingung für Rechtsideale wichtig.

Bezüglich des Durchschnittes der modularen maximalen Rechtsideale lautet das Problem 2 des Buches [2] von A. Kertész folgendermaßen:

Ist der Durchschnitt zweier modularer Rechtsideale eines Ringes stets modular?

Dieses Problem war für die Algebraiker auch früher bekannt, aber es wurde im Druck erst von Kertész [2] aufgeworfen.

Das Ziel dieser Arbeit ist nun zweifach: Einerseits geben wir einige Beispiele der Ringe an, in denen der Durchschnitt zweier modularer Rechtsideale nicht modular ist (Satz 1), und somit zeigt diese Lösung, daß die Antwort für das Problem 2 des Buches [2] von KFrTÉsz im allgemeiren »nein« ist. Andererseits betrachten

1 Nennt man ein Ideal $P$ eines Ringes primitiv (bzw. quasiprimitiv) im Ring $A$, wenn es ein modulares (quasimodulares) maximales Rechtsideal $R$ von $A$ mit $P=R: A$ gibt, so stimmt das Jacobsonsche Radikal nach Jacobson [1] (bzw. Verfasser [7]) mit dem Durchschnitt aller primitiven (bzw. quasiprimitiven) Ideale überein. Offenbar ist jedes primitive Ideal quasiprimitiv, und umgekehrt ist auch jedes quasiprimitive Ideal nach STEINFELD [5] und Verfasser [8] primitiv. In [8] gibt es eine Verschärfung des Resultates von [5]. Weiterhin stimmt das Jacobsonsche Radikal nach KERTÉsZ [3] mit $\Phi_{r}(A): A$ überein, wobei $\Phi_{r}(A)$ den Durchschnitt aller maximalen Rechtsideale des Ringes $A$ bezeichnet. 
wir einige, stärkere, hinreichende Bedingungen für einen Ring, die eine positive Antwort des Problems 2 von [2] in speziellen Ringen garantieren (Satz 3).

Die Lösung des Problems 2 des Buches [2] liefert ${ }^{2}$ der folgende

SATZ 1. Es gibt für jede endliche Mächtigkeit $2^{m \cdot n}(m \geqq 2, n \geqq 2)$ einen Ring $A$ mit $2 A=0$ und mit $2^{m \cdot n}$ Elementen derart, daß der Ring $m$ solche modulare Rechtsideale hat, aus denen der Durchschnitt beliebiger zweier modularer Rechtsideale nicht modular in $A$ ist. Weiterhin gibt es für jede unendliche Mächtigkeit $\aleph_{\alpha}$ einen Ring $A$ mit $2 A=0$ und mit $\aleph_{\alpha}$ Elementen derart, daß der Ring $\aleph_{\alpha}$ solche modulare Rechtsideale hat, aus denen der Durchschnitt beliebiger zweier modularer Rechtsideale nicht modular in $A$ ist.

BeweIs. Es seien $K_{2}$ der Primkörper mit zwei Elementen und $A$ die über $K_{2}$ durch die Elemente $t_{\alpha}$ erzeugte Algebra mit der Multiplikation

$$
t_{\alpha}^{k_{\alpha}} t_{\beta}^{k_{\beta}}=t_{\alpha}^{k_{\beta}}+t_{\beta}^{k_{\beta}}+t_{\alpha}^{k_{\alpha}+k_{\beta}} .
$$

Ist die Mächtigkeit der Menge der verschiedenen Symbole $t_{\alpha}$ eine endliche Zahl $m \geqq 2$, so gelte $t_{\alpha}^{n+1}=t_{\alpha}^{n}$ für jedes $t_{\alpha}$ mit einer festgewählten Zahl $n \geqq 2$. Ist aber die Mächtigkeit der Menge der verschiedenen Symbole $t_{\alpha}$ eine unendliche Mächtigkeit $\aleph_{\alpha}$, so dürfen entweder $t_{\alpha}^{n+1}=t_{\alpha}^{n}$ für jedes $t_{\alpha}$ mit einer festgewählten Zahl $n \geqq 2$ gelten, oder alle Potenzen von $t_{\alpha}$ für jedes $t_{\alpha}$ voneinander verschieden sein. Dann ist die Multiplikation in $A$ wegen $x+x=0$ für jedes $x \in A$ und wegen

$$
\begin{gathered}
t_{\alpha}^{k_{\alpha}}\left(t_{\beta}^{k_{\beta}} t_{\gamma}^{k_{\gamma}}\right)=t_{\alpha}^{k_{\alpha}}\left(t_{\beta}^{k \gamma}+t_{\gamma}^{k \gamma}+t_{\beta}^{k_{\beta}+k \gamma}\right)=t_{\alpha}^{k \gamma}+t_{\beta}^{k \gamma}+t_{\alpha}^{k_{\alpha}+k_{\gamma}}+t_{\alpha}^{k_{\gamma}}+t_{\gamma}^{k_{\gamma}}+t_{\alpha}^{k_{\alpha}+k \gamma}+ \\
+t_{\alpha}^{k_{\beta}+k_{\gamma}}+t_{\beta}^{k_{\beta}+k_{\gamma}}+t_{\alpha}^{k_{\alpha}+k_{\beta}+k_{\gamma}}=t_{\alpha}^{k_{\gamma}}+t_{\gamma}^{k_{\gamma}}+t_{\alpha}^{k_{\beta}+k_{\gamma}}+t_{\beta}^{k_{\gamma}}+t_{\gamma}^{k_{\gamma}}+t_{\beta}^{k_{\beta}+k_{\gamma}}+t_{\alpha}^{k_{\gamma}}+t_{\gamma}^{k_{\gamma}}+ \\
+t_{\alpha}^{k_{\alpha}+k_{\beta}+k_{\gamma}}=\left(t_{\alpha}^{k_{\beta}}+t_{\beta}^{k_{\beta}}+t_{\alpha}^{k_{\alpha}+k_{\beta}}\right) t_{\gamma}^{k_{\gamma}}=\left(t_{\alpha}^{k_{\alpha}} t_{\beta}^{k_{\beta}}\right) t_{\gamma}^{k_{\gamma}}
\end{gathered}
$$

offenbar assoziativ, und es gilt $\left(t_{\alpha}+t_{\beta}\right)^{2}=0$ für jedes $t_{\alpha}, t_{\beta}$. Weiterhin hat jedes Element des Ringes die Gestalt

$$
\sum_{i=1}^{k} f_{i}\left(t_{\alpha_{i}}\right)=\sum_{i=1}^{k} \sum_{j=1}^{k_{i}} a_{i j} t_{\alpha_{i}}^{j}
$$

wobei $f_{i}\left(t_{\alpha_{i}}\right)$ Polynome in $t_{\alpha_{i}}$, mit konstantem Glied 0 sind. Es gilt dabei dann und nur dann $f_{i}\left(t_{\alpha_{i}}\right)=f_{j}\left(t_{\alpha_{j}}\right)$ fül $t_{\alpha_{i}} \neq t_{\alpha_{j}}$, wenn $f_{i}\left(t_{\alpha_{i}}\right)=f_{j}\left(t_{\alpha_{j}}\right)=0$ ist. Wegen $x+x=0$ für jedes $x \in A$ erhält man $(1-x) A=(1+x) A$ für das modulare Rechtsideal $(1-x) A=$ $=[y-x y ; y \in A]$. Wegen $\left(1+t_{\alpha}\right) t_{\beta}=\left(1+t_{\alpha}\right) t_{\alpha}$ für jedes $t_{\beta}$ ergibt sich, daß das modulare Rechtsideal $\left(1-t_{\alpha}\right) A$ genau aus den Polynomen der Gestalt $\left(1+t_{\alpha}\right) f\left(t_{\alpha}\right)$ besteht, wobei das konstante Glied von $f\left(t_{\alpha}\right)$ verschwindet. Hiernach erhält man nach der vorigen Bemerkung gewiß

für $t_{\alpha_{i}} \neq t_{\alpha_{j}}$.

$$
\left(1-t_{\alpha_{i}}\right) A \cap\left(1-t_{\alpha_{j}}\right) A=0
$$

${ }^{2}$ Bezüglich der Resultate in Zusammenhang mit den Problemen 1 und 3 des Buches [2] von Kertész siehe die Arbeiten [6] und [9] des Verfassers. 
Dieser Durchschnitt, da er gleich Null ist, ist aber dann und nur dann modular im Ring $A$, wenn $A$ ein Linkseinselement hat. Wir werden aus der Voraussetzung, da $B$ der Ring $A$ ein Linkseinselement hat, einen Widerspruch ableiten.

Hat nämlich der Ring $A$ ein Linkseinselement $e$, so besitzt $e$ eine Gestalt

$$
e=\sum_{i=1}^{k} \sum_{j=1}^{s} a_{i j} t_{\alpha_{i}}^{j}
$$

Wegen $e t_{\sigma_{m}}=t_{\alpha_{m}}$ und $t_{\beta}^{l} t_{\alpha}=t_{\alpha}+t_{\beta}+t_{\beta}^{l+1}$ ergibt sich aus der Darstellung von $e$ nach einem Vergleich der Koeffizienten von $t_{\alpha_{m}}$ und $t_{\alpha_{i}}$ der folgende Widerspruch:

$$
1=\sum_{\substack{i=1 \\ i \neq m}}^{k} \sum_{j=1}^{n} a_{i j} ; \quad \sum_{\substack{j=1 \\ i \neq m}}^{n} a_{i j}=0
$$

woraus $1=0$ folgt, und somit folgt, daß $A$ kein Linkseinselement hat. Daher ist der Durchschnitt

$$
\left(1-t_{\alpha_{i}}\right) A \cap\left(1-t_{\alpha_{j}}\right) A=0
$$

für $t_{\alpha_{i}} \neq t_{\alpha j}$ gewiß nicht modular in $A$.

Die Mächtigkeit der modularen Rechtsideale der Gestalt $\left(1-t_{\alpha}\right) A$ ist entweder $m\left(\geqq 2,<\aleph_{0}\right)$ oder $\aleph_{\alpha}$. Weiterhin ist die Mächtigkeit der Elemente des Ringes $A$ entweder die endliche Zahl $2^{m \cdot n}(m \geqq 2, n \geqq 2)$, oder $\aleph_{\alpha}$.

Damit ist der Satz 1 bewiesen.

Bemirkung 2. Es kann erwähnt werden, daß der im Beweis des Satzes 1 betrachtete Ring $A$ für $m=2$ und $n=2$ dem durch die Matrizen

$$
M_{1}=\left(\begin{array}{ccccc}
0 & 1 & 0 & 0 & 0 \\
0 & 0 & 1 & 0 & 0 \\
0 & 0 & 1 & 0 & 0 \\
0 & 1 & 0 & 1 & 1 \\
0 & 1 & 0 & 1 & 1
\end{array}\right) ; \quad M_{2}=\left(\begin{array}{ccccc}
0 & 0 & 0 & 0 & 0 \\
0 & 1 & 1 & 1 & 0 \\
0 & 1 & 1 & 1 & 0 \\
0 & 0 & 0 & 0 & 1 \\
0 & 0 & 0 & 0 & 1
\end{array}\right)
$$

über dem Primkörper $K_{2}$ erzeugten Ring isomorph ist. In diesem Matrixring des Typs $5 \times 5$ gelten dann:

$$
\begin{gathered}
M_{1}^{2}=M_{1}^{3}=\left(\begin{array}{lllll}
0 & 0 & 1 & 0 & 0 \\
0 & 0 & 1 & 0 & 0 \\
0 & 0 & 1 & 0 & 0 \\
0 & 0 & 1 & 0 & 0 \\
0 & 0 & 1 & 0 & 0
\end{array}\right) ; \quad M_{2}^{2}=M_{2}^{3}=\left(\begin{array}{llllll}
0 & 0 & 0 & 0 & 1 \\
0 & 0 & 0 & 0 & 1 \\
0 & 0 & 0 & 0 & 1 \\
0 & 0 & 0 & 0 & 1 \\
0 & 0 & 0 & 0 & 1
\end{array}\right) \\
M_{1} M_{2}=\left(\begin{array}{lllll}
0 & 1 & 1 & 1 & 0 \\
0 & 1 & 1 & 1 & 0 \\
0 & 1 & 1 & 1 & 0 \\
0 & 1 & 1 & 1 & 0 \\
0 & 1 & 1 & 1 & 0
\end{array}\right) ; \quad M_{2} M_{1}=\left(\begin{array}{lllll}
0 & 1 & 0 & 1 & 1 \\
0 & 1 & 0 & 1 & 1 \\
0 & 1 & 0 & 1 & 1 \\
0 & 1 & 0 & 1 & 1 \\
0 & 1 & 0 & 1 & 1
\end{array}\right)
\end{gathered}
$$




$$
M_{1}+M_{2}=\left(\begin{array}{lllll}
0 & 1 & 0 & 1 & 0 \\
0 & 1 & 0 & 1 & 0 \\
0 & 1 & 0 & 1 & 0 \\
0 & 1 & 0 & 1 & 0 \\
0 & 1 & 0 & 1 & 0
\end{array}\right) \quad\left(\begin{array}{lllll}
0 & 1 & 1 & 0 & 0 \\
0 & 0 & 0 & 0 & 0 \\
0 & 0 & 0 & 0 & 0 \\
0 & 1 & 1 & 1 & 1 \\
0 & 1 & 1 & 1 & 1
\end{array}\right) ; \quad M_{2}+M_{2}^{2}=\left(\begin{array}{lllll}
0 & 0 & 0 & 1 & 1 \\
0 & 1 & 1 & 1 & 1 \\
0 & 1 & 1 & 1 & 1 \\
0 & 0 & 0 & 0 & 0 \\
0 & 0 & 0 & 0 & 0
\end{array}\right)
$$

Dieser Ring ist keine monomiale Algebra im Sinne von RÉDEI [4], und er hat folgende Multiplikationstabelle:

\begin{tabular}{|c|c|c|c|c|}
\cline { 2 - 3 } & $M_{1}$ & $M_{2}$ & $M_{1}^{2}$ & $M_{2}^{2}$ \\
\hline$M_{1}$ & $M_{1}^{2}$ & $M_{1}+M_{2}+M_{1}^{2}$ & $M_{1}^{2}$ & $M_{2}^{2}$ \\
\hline$M_{2}$ & $M_{1}+M_{2}+M_{2}^{2}$ & $M_{2}^{2}$ & $M_{1}^{2}$ & $M_{2}^{2}$ \\
\hline$M_{1}^{2}$ & $M_{1}^{2}$ & $M_{1}+M_{2}+M_{1}^{2}$ & $M_{1}^{2}$ & $M_{2}^{2}$ \\
\hline$M_{2}^{2}$ & $M_{1}+M_{2}+M_{2}^{2}$ & $M_{2}^{2}$ & $M_{1}^{2}$ & $M_{2}^{2}$ \\
\hline
\end{tabular}

Jetzt betrachten wir einige hinreichende Bedingungen, aus denen folgt, daß der Durchschnitt zweier modularer Rechtsideale des Ringes $A$ ebenfalls modular in $A$ ist.

Es gilt der

SAtz 3. Gilt eine der folgenden Bedingungen in einem Ring A, so ist der Durchschnitt von beliebigen zwei modularen Rechtsidealen von A ebenfalls modular in A:

a) $A$ hat ein Linkseinselement

b) Die Mengen $Q_{a}=[a+x-a x ; x \in A]$ und $Q_{b}=[b+y-b y ; y \in A]$ haben für jedes feste Paar der Elemente $a, b$ von A einen nichtleeren Durchschnitt, wobei $x, y$ alle Elemente von $A$ überlaufen.

ERGÄNZUNG 4. Wichtige Unterfälle sind, in welchen anstatt b) eine der folgenden Bedingungen erfüllt ist:

$\left.\mathrm{b}_{1}\right)$ Es gilt $a-b \in(1-a) A+(1-b) A$ für jedes $a, b \in A$;

$\mathrm{b}_{2}$ ) $A$ ist ein Radikalring im Sinne von JACOBSON [1];

$\mathrm{b}_{3}$ ) Für jedes $a, b \in A$ gibt es Elemente $q_{a} \in Q_{a}, q_{b} \in Q_{b}$ mit $q_{a} q_{b}=q_{b} q_{a}$;

$\left.\mathrm{b}_{4}\right) A$ ist kommutativ; 
$\mathrm{b}_{5}$ ) Gilt $q_{a} t_{a}=q_{a}$ für ein Element $q_{a} \in Q_{a}$ und für ein Element $t_{a} \in A$, so gilt auch $q_{b} t_{a}=q_{b}$ für ein Element $q_{b} \in Q_{b}$ für jedes $b \in A$;

$\left.\mathrm{b}_{6}\right) A$ hat ein Rechtseinselement;

$\mathrm{b}_{7}$ ) Es gilt $a-a b \in(1-a b) A$ für jedes Paar der Elemente $a, b \in A$.

Beweis des SAtzes 3. Hat $A$ ein Linkseinselement $e$, so ist wegen $(1-e) A=$ $=0 \subseteq R$ jedes Rechtsideal, und somit jeder Durchschnitt von Rechtsidealen modular in $A$.

Haben nun die Mengen $Q_{a}$ und $Q_{b}$ für jedes Element $a \in A, b \in A$ einen nichtleeren Durchschnitt, und bestehen $(1-a) A \subseteq R_{a}$ und $(1-b) A \subseteq R_{b}$ für die modularen Rechtsideale $R_{a}$ und $R_{b}$ von $A$, so erhält man $q_{a}=q_{b} \in Q_{a} \cap \bar{Q}_{b}$. Daher gibt es Elemente $x \in A, y \in A$ mit

woraus man

$$
q=a+x-a x=b+y-b y \in Q_{a} \cap Q_{b},
$$

$$
(1-q) A=(1-a)(1-x) A=(1-b)(1-y) A \subseteq(1-a) A \cap(1-b) A \subseteq R_{a} \cap R_{b},
$$

also die Modularität von $R_{a} \cap R_{b}$ in $A$ erhält.

BEWEIS DER ERGÄNZUNG 4.

$\mathrm{b}_{1}$ ) Gilt $a-b \in(1-a) A+(1-b) A$ für jedes $a \in A, b \in A$, so gibt es Elemente $x, y \in A$ mit

woraus

$$
a-b=(1-a)(-x)+(1-b) y
$$

folgt.

$$
q_{a}=a+x-a x=b+y-b y=q_{b} \in Q_{a} \cap Q_{b}
$$

$\mathrm{b}_{2}$ ) Ist $A$ ein Radikalring im Sinne von Jacobson, so gilt $(1-a) A=A$ für jedes $a \in A$, und somit gilt auch die Bedingung $\mathrm{b}_{1}$ ).

$\mathrm{b}_{3}$ ) Gibt es Elemente $q_{a} \in Q_{a}, q_{b} \in Q_{b}$ mit $q_{a} q_{b}=q_{b} \cdot q_{a}$ für jedes $a \in A, b \in A$, so gilt auch

$$
q_{a}+q_{b}-q_{a} q_{b}=q_{b}+q_{a}-q_{b} q_{a},
$$

woraus wegen der Assoziativität der Verknüpfung $x \circ y=x+y-x y$ offenbar folgt, daß $Q_{a} \cap Q_{b}$ nicht leer ist.

$\mathrm{b}_{4}$ ) Ist $A$ kommutativ, so gilt die Bedingung $\mathrm{b}_{3}$ ) und somit auch $\mathrm{b}$ ).

$b_{5}$ ) Bestehen gleichzeitig $q_{a}=q_{a} t_{a}$ und $q_{b}=q_{b} t_{a}\left(t_{a} \in A\right)$, so ergibt sich $t_{a}=q_{a}+t_{a}-q_{a} t_{a}=q_{b}+t_{a}-q_{b} t_{a}=a+\left(x+t_{a}-x t_{a}\right)-a\left(x+t_{a}-x t_{a}\right)=$ $=b+\left(y+t_{a}-y t_{a}\right)-b\left(y+t_{a}-y t_{a}\right)$ und somit gilt die Bedingung $\left.\mathrm{b}\right)$.

$\mathrm{b}_{6}$ ) Enthält $A$ ein Rechtseinselement $e$, so gilt wegen $q_{a} e=q_{a}$ und $q_{b} e=q_{b}$ die Bedingung $b_{5}$ ) und somit auch $\mathrm{b}$ ).

$\mathrm{b}_{7}$ ) Gilt $a-a b \in(1-a b) A$ für jedes Paar der Elemente $a \in A, b \in A$, so gibt es ein Element $c=c_{a, b} \in A$ mit $a-a b=c-a b c$. Daraus folgt $c=a-a b+a b c$. Mit der Bezeichnung $d=b-b c$ ergibt sich daher

$$
c=a-a d \text {. }
$$

Nun erhält man einerseits $a+d-a d \in Q_{a}$, andererseits $b+c-b c \in Q_{b}$, und wegen der Identität

$$
a+d-a d=a+(b-b c)-a d=b-b c+c
$$

auch $a+b-b c-a d \in Q_{a} \cap Q_{b}$, folglich die Gültigkeit der Bedingung b). 
Zum Schluß erwähnen wir einige offene Fragen:

Problem 1. Gibt es einen Ring $A$ ohne die Bedingungen a) und b), in dem der Durchschnitt zweier modularer Rechtsideale stets modular ist?

Problem 2. Ist der Durchschnitt zweier quasimodularer Rechtsideale in einem Ring stets quasimodular?

$\mathrm{Da}$ der in der Bemerkung 2 betrachtete Ring $A$ keinen von Null verschiedenen Rechtsannihilator enthält, ist der Durchschnitt $\left(1+M_{1}\right) A \cap\left(1+M_{2}\right) A$ ein quasimodulares (aber weder modulares noch maximales) Rechtsideal von $A$.

(Eingegangen am 4. März 1968.)

MTA MATEMATIKAI KUTATÓ INTÉZETE,

BUDAPEST, V., REÁLTANODA U. 13-15

\section{Literaturverzeichnis}

[1] N. JAcoBson, Structure of rings (Providence, 1956), Amer. Math. Soc. Coll. Publ. 37.

[2] A. KerTész, Vorlesungen über Artinsche Ringe (Budapest, 1968), Akadémiai Kiadó.

[3] A. Kertész, A characterization of the Jacobson radical, Proc. Amer. Math. Soc., 14 (1963), S. $595-597$.

[4] L. RÉDEI, Algebra I (Leipzig, 1959), Akad. Verlagsgesellschaft.

[5] O. Steinfeld, Eine Charakterisierung der primitiven Ideale eines Ringes, Acta Math. Acad. Sci. Hung., 19 (1968),S. $219-220$.

6] F. SzÁsz, Die Lösung eines Problems bezüglich einer Charakterisierung des Jacobsonschen Radikales eines Ringes, Acta Math. Acad. Sci. Hung., 18 (1967), S. 261-272.

[7] F. SzÁsz, Eine Charakterisierung des Jacobsonschen Radikales eines Ringes, Bull. Acad. Polon. Sci. Classe III, 15 (1967), S. 53-56.

[8] F. SzÁsz, The sharpening of a result concerning primitive ideals of an associative ring, Proc. Amer. Math. Soc., 18 (1967), S. 910-912.

[9] F. SzÁsz, Einige Kriterien für die Existenz des Einselementes in einem Ring, Acta Sci. Math. Szeged, 28 (1967), S. 31-37. 